$\Rightarrow$ KIDNEY CANCER

\title{
Tracing the steps of cancer evolution
}

Delineating the routes of tumour evolution that define clear cell renal cell carcinoma (ccRCC) could help to identify new treatment approaches and predict patient outcomes. Three papers recently published in Cell now reveal the interim findings of the TRACERx Renal study, which offer insights into the spatial and temporal evolutionary trajectories of RCC.

Following previous observations of extensive intratumour heterogeneity (ITH) within RCC, TRACERxRenal is aimed at elucidating the trajectories of tumour evolution that define RCC and explaining how they influence patient outcomes. "To capture the diversity within tumours, we performed very comprehensive sampling of the primary (or metastatic) tumour, taking up to 100 spatially separate biopsies from the surgical specimen," explains senior investigator Samra Turajlic. Following longitudinal sampling, genomic profiling was performed on each sample to determine the unique evolutionary pathway of each tumour.

In the first study, Turajlic et al. analysed 1,206 primary tumour regions from 101 patients and used conserved patterns of mutational ordering, timing, and co-occurrence to classify tumours into seven distinct evolutionary subtypes, or three broad

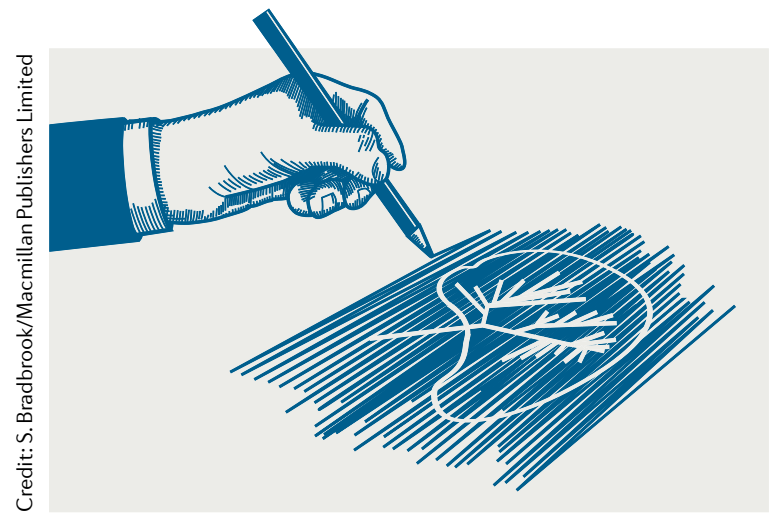

categories, which correlate with known clinical phenotypes. "The three categories are distinguished by two features - the number of chromosome alterations and the amount of genetic diversity," explains Turajlic. The first subtype - comprising aggressive tumours that rapidly grow and metastasize - acquires multiple mutational drivers and chromosomal alterations early in development, but has low ITH. Tumours that gradually accumulate chromosomal damage, which have high ITH, form the second subtype; they grow and metastasize slowly, often forming solitary or oligometastases. Tumours of the third subtype, defined by slow evolution with low ITH, acquisition of few genetic drivers, and low chromosomal instability, have limited metastatic potential. These insights illustrate the potential of such evolutionary classifications as biomarkers for guiding intervention and surveillance.

The second study investigated the features selected for in metastatic clones and the routes and timing of metastases across multiple anatomical sites. The analysis included a total of 575 primary and 335 matched metastatic biopsy samples from 100 patients enrolled in TRACERx Renal and 2 validation cohorts. "The clonal populations that are metastasis-competent have high levels of chromosomal damage and, in particular, are enriched for loss of chromosome 9p and 14q," explains Turajlic. Interestingly, metastases contained fewer subclonal driver alterations than their matched primary tumours, suggesting that metastatic tumours are more homogeneous. In addition, two distinct modes of metastatic dissemination were uncovered. Primary tumours with low ITH and high genomic instability acquired metastatic competence from the most recent common ancestor, leading to rapid progression, whereas those with low genomic instability and high ITH gradually acquired metastatic capacity, leading to attenuated progression. Importantly, the data suggest that metastasis-enabling events (such as $9 \mathrm{p}$ and/or 14q loss) could be potential predictive biomarkers.

The third study traced the early events leading to RCC in 95 biopsy samples from 33 patients. The most interesting finding was that the initiating driver abnormality, chromosome 3 p loss with $5 q$ gain, occurs in childhood and adolescence, $30-50$ years before diagnosis. Clonal expansion following chromosome $3 p$ loss results in only a few hundred cells, which, upon acquisition of somatic driver mutations, might lead to sporadic tumours. The authors postulate that the well-defined sequence and timing of these initiating events could lead to new opportunities for early intervention.

Overall, these interim findings shed light on the deterministic evolutionary pathways in RCC and could lead to new options for patient management. The next milestone for TRACERx Renal will be the completion of accrual ( $>300$ patients). "Meanwhile, we are looking at other aspects of clinical behaviour such as response to drug therapy in relation to these evolutionary subtypes," concludes Turajlic. "We are also planning the next steps to validate this approach as a clinical tool for routine use to inform prognosis and help clinical decision-making."

Conor A. Bradley

Originally published in Nat. Rev. Clin. Oncol. (https://doi.org/10.1038/s41571-018-0033-z).

ORIGINAL ARTICLES Turajlic, S. et al. Deterministic evolutionary trajectories influence primary tumor growth: TRACERx Renal. Cell 173, 595-610 (2018) | Turajlic, S. et al. Tracking cancer evolution reveals constrained routes to metastases: TRACERx Renal. Cell 173, 581-594 (2018) | Mitchell, T. J. et al. Timing the landmark events in the evolution of clear cell renal cell cancer. TRACERx Renal. Cell 173, 611-623 (2018) 